\title{
The role of neuraminidase inhibitors in the treatment and prevention of influenza
}

\author{
Naem Shahrour* \\ American International Health Council, 414 South Craig St. \#269, Pittsburgh, PA 15213, USA
}

The causative agents of acute respiratory infections in children and adults are mostly thought to be viruses. Many types of viruses could cause similar symptoms of ARI. Among them, influenza viruses $A$ and $B$ and respiratory syncytial virus (RSV) are thought to be the most important because of the severity of illness after infection and their high communicability in the human population [1]. Type $\mathrm{C}$ influenza virus usually is not serious and typically not included in discussion about influenza infection [2]. In this article, discussion is limited to influenza A and B only. These viruses belong to the orthomyxoviridae family of viruses [3].

Influenza infects an estimated 120 million people in the United States, Europe, and Japan each year and is a major cause of morbidity and mortality. In periods of flu pandemics, infection rates could be even higher. In the United States, out of an estimated 20 million cases of flu each year, up to 40000 Americans die, and more than 400000 are hospitalized. The resulting economic burden has been estimated to be as high as $\$ 12$ billion. In the last few years, new developments in the prevention, diagnosis, and treatment of influenza have been introduced that will change the clinical milieu in the new millennium.

The traditional vaccine approaches to the prophylaxis have been problematic owing to the ability of these viruses to undergo antigenic shift by exchanging genomic segments or by undergoing antigenic drift, consisting of point mutations in the surface glycoproteins haemagglutinin (HA) and neuraminidase (NA) genes as a result of an error-prone viral polymerase. Antigenic shift is seen only in influenza A viruses and occurs when a novel HA or NA subtype is introduced from an animal influenza virus reservoir into the human population. Antigenic drift occurs in influenza A and B in and around the antibody binding site. Therefore, the annual update of influenza is necessary because of the annual occurrence of antigenic drifts. For example, for the 1999-2000 season, it was recommended that vaccines contain an A/Sydney/5/97 (H3N2)-like virus, an A/Beijing/262/95 (H1N1)-like virus, and a B/Beijing/184/93-like virus or B/shangdong/7/97-like virus. However, significant morbidity and mortality were clinically observed during this season, and indicated the obvious limitations of this method of prevention. As a result of these ongoing observations, other prevention and treatment modalities have been investigated and some have already been introduced to practice. The most recent breakthrough in that direction is the introduction of neuraminidase inhibitors for the treatment and prevention of influenza infection.

The neuraminidase is a surface glycoprotein that is composed of eleven conserved residues [4] which catalyze the cleavage of sialic acid residues terminally linked to glycoproteins and glycolipids and plays an important role in the replication of the virus. A recent new study demonstrated that Tyr409 is the most critical residue for enzyme activity, and that Asp149, Arg223, Glu275, and Arg374 also play important roles in enzyme catalysis [2]. Neuraminidase function is critical for the spread of virus to new cells, and if the enzyme activity is inhibited, then virus infection is abrogated $[2,5]$. Also, there is evidence that this enzyme plays some role in the introduction of apoptosis to the infected cells [6].

Currently, there are two neuraminidase inhibitors (NI) available for clinical use: zanamivir and oseltamivir. In phase II trials (NAIB2005 and NAIB2008), benefit was seen in early treatment (within 36 hours of onset of illness). Virological substudies showed mean reductions in virus shedding after 24 hours of treatment of 1.5 to $2.0 \log$ [10] $50 \%$ tissue culture infective doses compared with a placebo, with no reemergence of virus after completion of the therapy $[7,8]$. In phase III trials, median times to alleviation of major symptoms were reduced by up to 2.5 days after treatment. Benefit of treatment in terms of time to return to normal activities, reductions in consumption of acetaminophen, and the reductions in the level of interference of influenza with sleep, work, leisure, and recreational activities were reported [9].

In the prophylaxis trials, statistically significant reductions in the incidence of influenza A was reported with the use of inhaled zanamivir in a double blind study that included 1107 persons in two university communities $[9,10]$. In this study, zanamivir was $67 \%$ efficacious in preventing laboratory-confirmed clinical influenza and $84 \%$ efficacious in preventing laboratory-confirmed illnesses with fever. Also, intravenous zanamvir (600 mg twice daily for 5 days) used 4 hours prior to intranasal inoculation with infectious doses of influenza A virus was successful in reducing the frequency of viral shedding ( $0 \%$ versus $100 \%$ in placebo), seroconversion ( $14 \%$ versus $100 \%$ in placebo), fever (14\% versus 
$88 \%$ in placebo), upper respiratory tract illness ( $0 \%$ versus $100 \%$ in placebo), and total symptom score (1\% versus $44 \%$ in placebo) [11]. Another trial showed that outbreak of influenza A and B were prevented in a nursing home community [9].

Oral administration of oseltamivir (GS4104) in two randomized trials (1559 subjects given $75 \mathrm{mg}$ once or twice daily) [12] reduced the risk of infection during the influenza season from $4.8 \%$ in placebo to $1.2 \%$ and $1.3 \%$ in the treatment groups. The protective effects of the drug were $74 \%$ in all study sites combined and $84 \%$ in the areas where the rate of influenza was higher. Another smaller study reached similar conclusions [13].

The coadministration of NI with inactivated trivalent influenza vaccine was investigated in England. It was found that it does not adversely affect the production of antihaemagglutinin antibodies in the serum [14]. Finally, although fighting influenza seems like boxing a heavyweight champion and odds are you will end up flat on your back, but with proper precautions and the development of new drugs you may be able to avoid getting knocked out.

\section{REFERENCES}

[1] Shigeta S. Approaches to antiviral chemotherapy for acute respiratory infections. Antivir Chem Chemother. 1998;9(2):93-107.

[2] Ghate AA, Air GM. Site-directed mutagenesis of catalytic residues of influenza virus neuraminidase as an aid to drug design. Eur J Biochem. 1998;258(2):320-331.

[3] Colacino JM, Staschke KA, Laver WG. Approaches and strategies for the treatment of influenza virus infections. Antivir Chem Chemother. 1999;10(4):155-185.

[4] Finley JB, Atigadda VR, Duarte F, et al. Novel aromatic inhibitors of influenza virus neuraminidase make selective interactions with conserved residues and water molecules in the active site. J Mol Biol. 1999;293(5):1107-1119.

[5] Kim CU, Chen X, Mendel DB. Neuraminidase inhibitors as anti-influenza virus agents. Antivir Chem Chemother. 1999;10(4):141-154.
[6] Morris SJ, Price GE, Barnett JM, et al. Role of neuraminidase in influenza virus-induced apoptosis. J Gen Virol. 1999;80(pt1):137-146.

[7] Barnett JM, Cadman A, Gor D, et al. Zanamivir susceptibility monitoring and characterization of influenza virus clinical isolates obtained during phase II clinical efficacy studies. Antimicrob Agents Chemother. 2000;44(1):78-87.

[8] The MIST (Management of Influeneza in the Southern Hemisphere Trialists) Study Group. Randomized trial of efficacy and safety of inhaled zanamivir in treatment of influenza A and B virus infection. Lancet. 1998;352(9144):1877-1881.

[9] Dunn CJ, Goa KL. Zanamivir: a review of its use in influenza. Drugs. 1999;58(4):761-784.

[10] Monto AS, Robinson DP, Herlocher ML, et al. Zanamivir in the prevention of influenza among healthy adults: a randomized controlled trial. JAMA. 1999;282(1):31-35.

[11] Calfee DP, Peng AW, Cass LM, et al. Safety and efficacy of intravenous zanamivir in preventing experimental human influenza A virus infection. Antimicrob Agents Chemother. 1999; 43(7):1616-20.

[12] Hayden FG, Atmar RL, Schilling M, et al. Use of the selective oral neuraminidase inhibitor oseltamivir to prevent influenza. N Engl J Med. 1999;341(18):1336-1343.

[13] Hayden FG, Treanor JJ, Fritz RS, et al. Use of the oral neuraminidase inhibitor oseltamivir in experimental human influenza: randomized controlled trials for prevention and treatment. JAMA. 1999;282(13):12401246.

[14] Webster A, Boyce M, Edmundson S, et al. Coadministration of orally inhaled zanamivir with inactivated trivalent influenza vaccine does not adversely affect the production of antihaemagglutinin antibodies in the serum of healthy volunteers. Clin Pharmacokinet. 1999;36(suppl 1):51-58.

\footnotetext{
* E-mail: aihc@asca.com

Fax: +1 412 4418198; Tel: +1 4124418358
} 

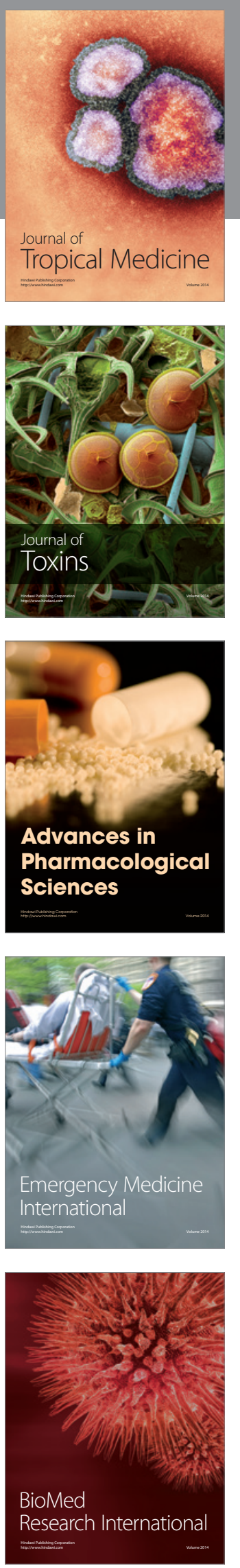
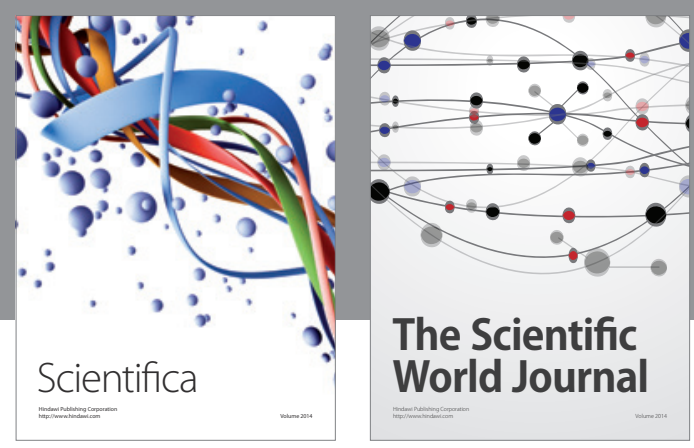

The Scientific World Journal
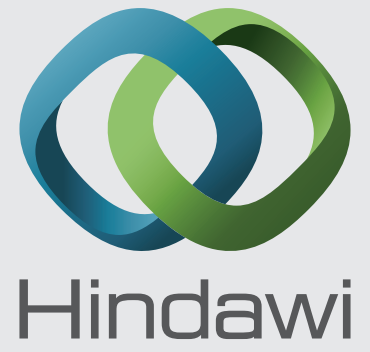

Submit your manuscripts at

http://www.hindawi.com
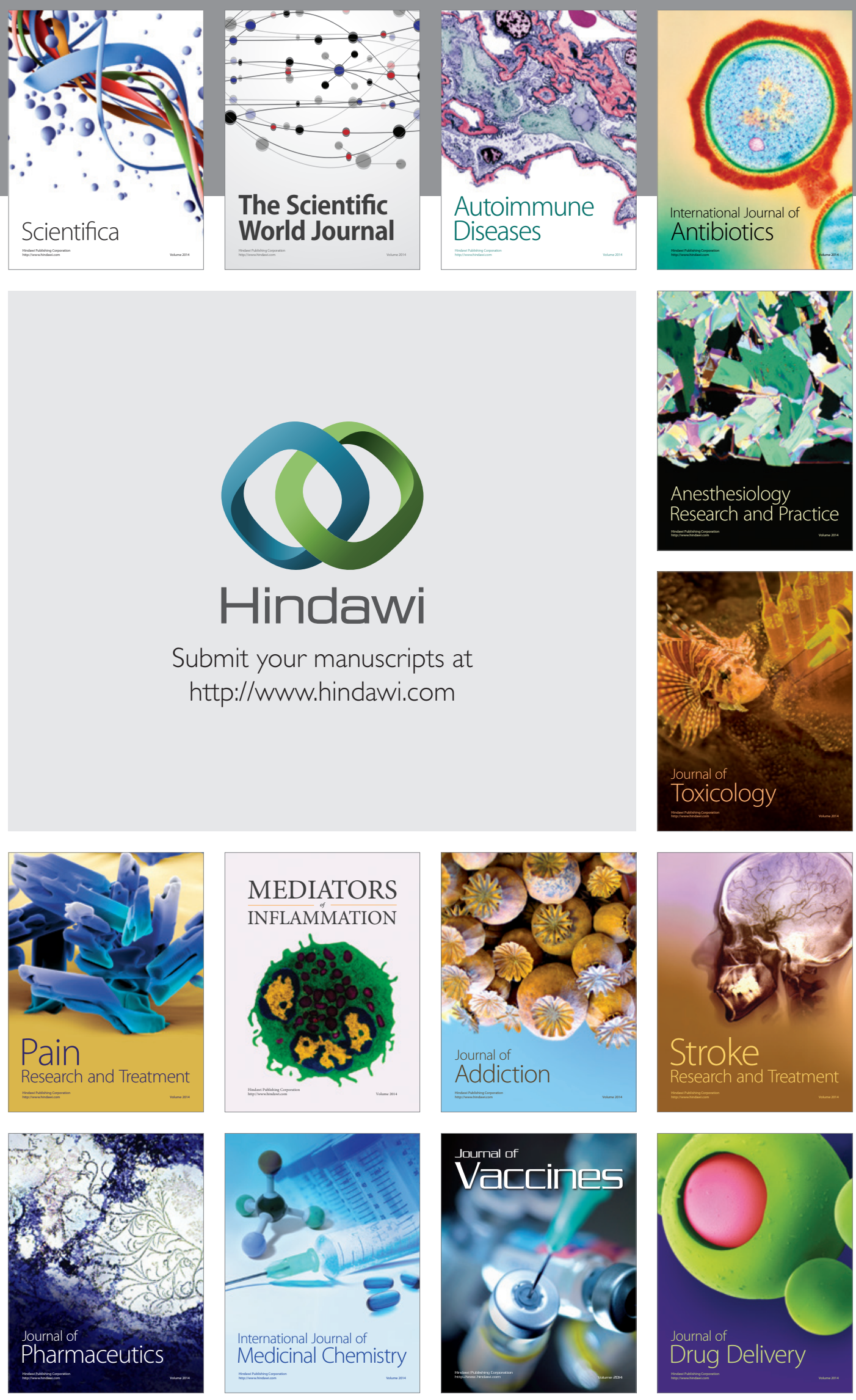